\title{
PORRESPONDENCE
}

\section{The Pharmacological Treatment of Arterial Hypertension in Frail, Older Patients}

\author{
A Systematic Review \\ by Viktoria Mühlbauer, PD Dr. med. Dhayana Dallmeier, Ph.D., Simone Brefka, Claudia Bollig, M.Sc., \\ Dr. Sebastian Voigt-Radloff, M.Sc., and Prof. Dr. med. Michael Denkinger in issue 3/2019
}

\section{Depressing Conclusion}

We have to thank Ms. Mühlbauer and her coworkers for dedicating their review (1) to a topic of enormous relevance in daily practice. Their conclusion is - almost as expected - depressing. Due to the lack of evidencebased information about this problem, individual decisions have to be made on a case-by-case basis (1). However, we do know that in frail, older patients it rather does more good than harm to the patient if a medication can be avoided.

However - and this is my criticism-the effect measures listed in the extensive Tables $1 \mathrm{a}$ and $1 \mathrm{~b}$ are exclusively stated as hazard ratios, i.e. as relative risks (RR), which is not very helpful.

Here an example: In Table 1a, a HR $=0.75$ is stated under HYVET for stroke in the first row.

This can mean that in the placebo group $6.30 \%$ and in the active group $5.05 \%$ of patients experienced a stroke. Consequently, one would arrive at the following figures:

- Absolute risk reduction $(\mathrm{ARR})=1.25 \%$

$(6.30-5.05)$

- Number needed to treat $(\mathrm{NNT})=80(100: 1.25)$

- Relative risk reduction $(\mathrm{RRR})=25 \%$

(1.25 based on 5.05)

- Relatives risk $(\mathrm{RR})=75 \%$

- Hazard ratio $(\mathrm{HR})=0.75$.

However, one would also arrive at $\mathrm{HR}=0.75$ if with placebo $63 \%$ and with active treatment $50.5 \%$ of patients had experienced a stroke (consequently: $\mathrm{ARR}=12.5 \%, \mathrm{NNT}=8$ ) or if with placebo only $0.63 \%$ and with active treatment $0.505 \%$ of patients had experienced a stroke (consequently: $\mathrm{ARR}=0.125 \%$, $\mathrm{NNT}=800$ ).

These considerations show that relative values (relative risks, hazard ratios, odds ratios, relative risk reductions) cannot reliably be interpreted and thus are unsuitable for the evaluation of effects.

DOI: 10.3238/arztebl.2019.0346a

\section{References \\ 1. Mühlbauer V, Dallmeier D, Brefka S, Bollig C, Voigt-Radloff S, Denkinger M: The pharmacological treatment of arterial hypertension in frail, older patients—a systematic review. Dtsch Arztebl Int 2019; 116: 23-30.}

In Reply:

We would like to thank Prof. Meyer for his valuable comments on our article.

We agree that, besides reporting relative risks and hazard ratios, it is necessary to also report the absolute risk reduction (ARR) and the number needed to treat (NNT) to allow a clinical evaluation of treatment effects.

In our systematic review (1), we had to rely on information from the primary studies which was mostly inadequate for ARR/NNT, as for example in HYVET. Furthermore, NNT is strongly influenced by the baseline risk; thus, it cannot be considered a proper approach to compare NNT between the strata frail versus non-frail.

If one looks only at frail patients (frailty index $>0.21$ ) in the SPRINT study, one will find an absolute risk reduction (ARR) of $4.9 \%$ and an NNT of 20. Among non-frail patients, ARR was $3.3 \%$ with an NNT of 30.

At what point the difference between NNTs should be considered as relevant has to be discussed in the clinical context. As our primary aim was to compare the evidence for different treatments of arterial hypertension in frail versus non-frail older patients, calculation of NNT (or the "number needed to harm" $[\mathrm{NNH}]$ ) was not one of our main goals. Therefore, we presented the studies that had assessed effect modification by physical frailty. Unfortunately, however, the currently available data is not sufficient to warrant a differential treatment of arterial hypertension.

DOI: 10.3238/arztebl.2019.0346b

\section{References}

1. Mühlbauer V, Dallmeier D, Brefka S, Bollig C, Voigt-Radloff S Denkinger $\mathrm{M}$ : The pharmacological treatment of arterial hypertension in frail, older patients-a systematic review. Dtsch Arztebl Int 2019; 116: 23-30.

\section{Corresponding authors}

Prof. Dr. med. Michael Dieter Denkinger

PD Dr. med. Dhayana Dallmeier, Ph.D.

Geriatrie der Universität UIm

and Geriatrisches Zentrum Ulm/Alb-Donau

Agaplesion Bethesda Klinik, Ulm, Germany

forschung@bethesda-ulm.de

Conflict of interest statement

The authors of both contributions declare that no conflict of interest exists 